\title{
Estado actual de la educación ambiental en un contexto escolar
}

\author{
Luis Guillermo González Merchán* \\ Cesar Olimpo Melo Melo** \\ Gustavo Adolfo Flórez Restrepo***
}

Artículo de Reflexión

Fecha de Recepción: 20 junio 2018.

Fecha de Aprobación: 16 octubre 2018.

\section{Resumen}

El trabajo se desarrolló en el Instituto Técnico La Nueva Familia de Duitama y propone como eje de intervención la dinamización del Proyecto Ambiental Escolares (PRAE) como estrategia para integrar saberes y prácticas de la educación ambiental. Se estableció la caracterización de los alcances del proyecto ambiental escolar a partir de los saberes y prácticas de la comunidad educativa. La investigación fue cualitativa fundamentada en investigación acción, se establecieron dos categorías, Ambiente y Proyecto Ambiental Escolar, se construyeron los instrumentos de indagación y se analizó su incidencia en la cultura ambiental escolar.

Se evidenció que las prácticas están habitualmente asociadas al desarrollo de la Educación Ambiental sobre la visión naturalista y conservacionista y que por tanto desplaza los procesos de interacción socio-cultural en la formación de los niños y jóvenes, sumado a esto se reconoce que si bien se han desarrollado acciones en pro de la educación ambiental esta no ha logrado incidir de manera sistemática en los procesos de planeación y desarrollo institucional, sino por el contrario en algunas actividades muy puntuales y esporádicas, lo que fundamenta la importancia de incidir en darlo un nuevo sentido al PRAE y movilizar una cultura ambiental en la comunidad educativa.

Palabras clave: cultura, educación ambiental, ambiente, entorno, saberes.

\footnotetext{
* Instituto Técnico Nueva

Familia Duitama

Boyacá - Colombia guillermogonzalez_m@ hotmail.com

** Instituto Técnico Nueva

Familia Duitama

Boyacá - Colombia cesome68@yahoo.com

*** Universidad Pedagógica y Tecnológica de Colombia Boyacá - Colombia guadflo@gmail.com
}

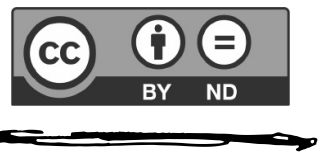




\section{Introducción}

El Instituto Técnico Nueva Familia, cuenta entre sus estudiantes con jóvenes de estratos uno y dos del SISBEN, de la ciudad de Duitama, además de una población flotante del resto del país, evidenciándose problemas de comportamiento frente al cuidado y conservación del entorno, hecho que se refleja principalmente en el desaseo de aulas, áreas comunes, destrucción de los bienes materiales de la institución y generación y ausente clasificación de los residuos sólidos, estos comportamientos evidencian una limitada cultura ambiental en la comunidad educativa. Desde esta perspectiva la educación ambiental contribuirá a desarrollar el sentido de responsabilidad y solidaridad como base de un nuevo orden nacional para garantizar la conservación, preservación y el mejoramiento del ambiente.

El comportamiento de los estudiantes dentro de las instalaciones de una institución educativa son un fiel reflejo del comportamiento frente al cuidado y preservación del entorno y todas sus dinámicas, sin embargo, se presenta

El Instituto Técnico Nueva Familia, cuenta entre sus estudiantes con jóvenes de estratos uno y dos del SISBEN, de la ciudad de Duitama, además de una población flotante del resto del país, evidenciándose problemas de comportamiento frente al cuidado y conservación del entorno, hecho que se refleja principalmente en el desaseo de aula

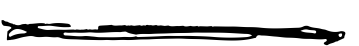

quinto y sexto e indirectamente espera incidir en toda la institución, por lo que puede aportar al mejoramiento de la educación y contribuir a que las comunidades educativas tengan una mayor conciencia ambiental, que conlleve a una relación más armoniosa con los procesos naturales propios de su entorno y así aportar a la solución de los problemas ambientales desde una ética del cuidado y promoción del desarrollo humano.

De igual manera, las instituciones educativas en su estructura curricular, el Proyecto Educativo Institucional (PEI) y los proyectos pedagógicos transversales hacen énfasis en que los estudiantes deben construir y aplicar conocimientos, diseñar métodos, estrategias e instrumentos para trasformar la realidad próxima al servicio de la humanidad, profundizando los saberes en Ciencias Naturales, participando creativamente en la clasificación de valores que faciliten la convivencia social $y$ dignifiquen la existencia humana.

Por lo tanto, es necesario diseñar y desarrollar estrategias desde el proyecto ambiental escolar, que permitan el fortalecimiento de la cultura ambiental, siendo uno de los primeros momentos el caracterizar los alcances del proyecto ambiental escolar a partir de los saberes y prácticas de la comunidad educativa, para luego diseñar e implementar estrategias desde el PRAE y finalmente establecer los alcances de las estrategias implementadas desde este proyecto.

De esta manera este proyecto se fundamentó en los procesos de 
dinamización del PRAE como estrategia que fortalecerá la cultura ambiental en la Institución Educativa y como mecanismo para integrar a diferentes actores sociales desde la realidad ambiental del entorno que comparten.

\section{Sustentos Teóricos}

La investigación realizada por Pérez (2013) titulada: "Comportamiento ambiental en estudiantes de secundaria", tuvo como propósito abordar el problema del comportamiento asumido por los estudiantes de secundaria de la Escuela Básica Nacional "José Cortés de Madariaga”, en relación con el estudio del medioambiente y la necesidad de lograr una calidad ambiental en esa Institución. Evidenciando una serie de fallas en cuanto a la conservación, el mantenimiento y el cuidado de su medioambiente. El personal docente encuestado expreso que la problemática del comportamiento ambiental de sus alumnos hacia el medioambiente escolar es un grave problema para la institución y los que ahí conviven, lo que se evidencio en las acciones realizadas por la población estudiantil entre ellos rayar paredes, no cuidar los pupitres, dañar las griferías de los baños, tirar la basura en el piso y, no tienen la precaución de cerrar la llave del agua. Parte de las conclusiones de este trabajo es señalar la misión de la Escuela en función de orientar a los Padres y Representantes, apoyando su formación para que transmitan a sus hijos o representados los valores ambientales, dando cumplimiento de esta manera a la función ecológica que debe ejercer la Familia. Este trabajo le aporta a la presente investigación, ya que establece que el deterioro por el mal trato de los recursos naturales y artificiales que forman el medioambiente escolar, es cuestión de fallas en la formación de valores y principios del hogar.

Arango (2015) en el trabajo titulado Sentidos de la Educación Ambiental para las Formadoras de la Primera Infancia en el Núcleo Educativo 915, tuvo por objetivo interpretar los sentidos que le dan a la Educación ambiental las formadoras de la primera infancia en el Núcleo educativo 915 de la ciudad de Medellín, por medio de una metodología cualitativa de carácter comprensivo. Se concluyó que los sentidos de educación ambiental que tienen las formadoras de la primera infancia en el Núcleo 915 de Medellín, se definen en dos tendencias; la primera corresponde a una visión Conservacionista/recordista, donde se concibe al ambiente como los recursos que están a disposición de los seres humano para su supervivencia y la educación ambiental es el proceso orientado a la conservación y cuidado de dichos recursos, para garantizar su permanencia y disposición. La segunda tendencia corresponde a la visión sistémica, que concibe al ambiente como un sistema dinámico, donde sus componentes (político, natural, social, económico, cultural) se interrelacionan y la educación ambiental está orientada a que los individuos y comunidades se apropien de la realidad de su contexto, comprendan su interdependencia del ambiente y lo valoren y respeten. 
Este trabajo le aporta a la presente investigación, ya que es un aporte a la reflexión y análisis del estado de la educación ambiental que se está impartiendo actualmente, a los niños y niñas en su primera infancia y en general en las instituciones educativas públicas.

Barrera, (2014) en el trabajo Determinación del nivel de conocimiento ambiental de los estudiantes de básica secundaria y media en las instituciones educativas públicas del municipio de Manizales, cuyo objetivo fue determinar nivel de conocimiento ambiental de los estudiantes de básica secundaria y media. Se concluyó que en el nivel de conocimiento de los estudiantes de último grado de básica secundaria y media del municipio de Manizales no se evidencia la aplicación de la normatividad ambiental para el desarrollo de los Proyectos Ambientales Escolares, que son medibles bajo la metodología de las pruebas "Saber", mediante la aplicación de los estándares básicos de competencias; solo el 45\% de los estudiantes aprueban el cuestionario de conocimiento ambiental. Este trabajo le aporta a la presente investigación, ya que es un punto de partida para determinar el nivel de conocimiento ambiental de los estudiantes objeto de investigación.

Rondón (2016) en el trabajo titulado Percepciones socio ambientales en las comunidades estudiantiles de dos instituciones educativas dentro del contexto rural y urbano, hacia el PNN ranchería en Paipa Boyacá, a través de educación ambiental tuvo por objetivo interpretar las percepciones socio ambientales en las comunidades estudiantiles de dos instituciones educativas desde el contexto urbano y rural, frente al Parque Natural Municipal Ranchería y proponer estrategias desde la educación ambiental para la adopción de buenas prácticas ambientales. Se concluyó que la disposición encontrada en los estudiantes de ambas instituciones educativas para vincularse en actividades en pro de su medio ambiente, es una excelente oportunidad para emplear estrategias de educación ambiental enfocadas directamente en los aspectos que más preocupación generan en la comunidad, como lo son los cuerpos de agua y a partir de ahí ejecutar acciones para su conservación y rehabilitación. Parte de las afirmaciones de este trabajo incluye el considerar la educación ambiental como pilar para sembrar las bases de una cultura ambiental tendiente a la sostenibilidad, los estudiantes presentan dificultades al reconocer el papel fundamental de la misma y la asocian muchas veces con charlas ambientalistas. Este trabajo le aporta a la presente investigación ya que establece que se requiere innovar, sensibilizar, concientizar, materializar y difundir la educación ambiental de las instituciones educativas.

Torres (2011), en su trabajo titulado, Medio ambiente y Proyecto Ambiental Escolar (PRAE) en el Colegio Nicolás Esguerra, realizo un estudio teórico sobre el medio ambiente, la evolución de la educación ambiental, las problemáticas ambientales específicas 
de la localidad 8-Kennedy y sobre proyectos ambientales relevantes. Tuvo por objetivos proponer y presentar a los docentes de la institución una metodología de trabajo escolar, orientada a la construcción del PRAE y a precisar el trabajo de aula que les compete, con el fin de propiciar la toma de decisiones colegiada. Se concluyó que debido a la crisis actual que afronta el planeta, uno de los proyectos educativos más importantes es el PRAE. Este proyecto se constituye en un reto trascendental para las instituciones educativas, ya que lo deben llevar a cabo de una manera consciente y comprometida, evitando asumirlo como un trabajo escolar que se realiza para cumplir los requerimientos gubernamentales. Entre las conclusiones se encuentra que los docentes son los llamados a gestionar los procesos de evolución, adaptación y desarrollo dentro de la escuela, por tanto, les compete el deber social de liderar, acompañar, enriquecer y evaluar constantemente las acciones educativas, y la puesta en marcha de nuevos proyectos. Este trabajo le aporta a la presente investigación ya que establece que debe generarse un cambio en los esquemas de pedagogía tradicional, construyendo un proyecto que no solo involucre a la escuela sino a cada persona como ser vivo. Se establece que corresponde asumir posturas activas, renunciando a la indiferencia ante una serie de problemáticas ambientales que están incidiendo a nivel social, cultural y económico en todo el mundo.

Duque Serna, (2015) en su trabajo de maestría titulado Educación ambiental de estudiantes, docentes $y$ directivas de educación básica secundaria. Se reconocieron las políticas administrativas que inciden en el desarrollo de la educación ambiental según docentes, estudiantes y directivos del grado noveno de dicha institución. Se concluyó que la educación ambiental influye en los diferentes actores de la institución educativa, como son los estudiantes, docentes y directivas, por cuanto ha generado preocupación, frente al cuidado, conservación, protección, preservación y manejo de los recursos naturales del medio ambiente en general, en especial lo concerniente a la zona de influencia donde está ubicado el Liceo Taller San Miguel.

\section{Teorías del conocimiento}

\section{Educación ambiental}

Laeducación ambientalcentrasu estudio en la comprensión y sensibilización que tienen los sistemas naturales, que requieren protección y conservación para las futuras generaciones. Dentro de esta premisa se ha ubicado a la ecología como ciencia natural y deja de lado las interacciones de esta con otras áreas del conocimiento, la tecnológica que se ha enfatizado en el análisis de los problemas ambientales como un producto del desarrollo tecnológico, la economía ve como valor de cambio a los recursos naturales siendo independiente de las relaciones que ocurren entre las sociedades y estos recursos, prevaleciendo en ella el concepto de crecimiento económico y una que otra tendencia política que
Duque Serna, (2015) en su trabajo de maestría titulado Educación ambiental de estudiantes, docentes $y$ directivas de educación básica secundaria. 
hacen análisis y reflexiones a partir de la satisfacción de las necesidades de los seres humanos (Ángel A, 1992).

Además la educación ambiental está inmersa en la cultura escolar y se manifiesta cuando trata de valorar, transmitir o interpretar la cultura social (currículo escolar), es decir, la cultura escolar convierte la cultura social en currículo por medio de las escuelas y sus profesores (mediadores) (Da Silva, 2001).

El acceso a la cultura ambiental se concreta a partir de los PRAE que según Da Silva (2001), la posición de la escuela y la educación frente a la cultura se puede situar en tres dimensiones: La escuela como transmisora crítica de la cultura, La escuela reproductora de la cultura y La escuela liberadora en la cultura. Por esto, en los últimos años se ha buscado impulsar estrategias y actividades que ayuden a resolver la problemática ambiental. Al respecto Rengifo B. 2012 expresa en este sentido que se debe promover que las personas adopten modos de vida que sean compatibles con la sostenibilidad adquirida, mediante la adecuada exploración, explotación, utilización, y manejo de los recursos naturales.

Con respecto a los objetivos de la educación ambiental, uno de ellos es que los individuos y las comunidades deben comprender la complejidad del ambiente natural y el creado por el ser humano, resultado de este último de la interacción de los factores biológicos, físico-químicos, sociales, económicos, políticos y culturales para que se adquieran los conocimientos, valores, actitudes, destrezas y habilidades que les permitan participar de manera responsable, ética, afectiva en la previsión de la problemática en los cambios climáticos; desde esta perspectiva la educación ambiental contribuirá a desarrollar el sentido de responsabilidad y solidaridad como base de un nuevo orden nacional para garantizar la conservación, preservación y el mejoramiento del ambiente (Rengifo 2012).

En un evento internacional importante en torno a la Educación Ambiental ha sido la Conferencia Intergubernamental sobre Educación Ambiental (TbilisiURSS, octubre de 1977). En Tbilisi se crea el conjunto o recopilación teórica de la Educación Ambiental, desde su definición hasta la determinación de sus áreas de actuación (Calvo, 1997). En razón a lo anterior, el gran objetivo fue que se considerara Educación Ambiental como pieza indiscutible en la educación permanente que todo ciudadano necesita para convivir responsablemente con el entorno físico y natural, humano y social, de allí surge la exigencia de reafirmarse la educación ambiental como una práctica educativa concienciadora (Caride \& Meira, 2001).

\section{Construcción de Cultura Ambiental}

Se tiene la posibilidad de pensar la cultura no solo como el espacio de la reproducción social sino como el espacio privilegiado para la innovación y la resistencia, según Bourdieu citado en Reguillo (2001). 
La forma de analizar la interrelación entre el hombre y el ambiente requiere de un nuevo enfoque, además de la estructuración de iniciativas y proyectos de investigación que llamen la atención de los profesionales y estudiantes, que propendan por la conservación y mejoramiento de la calidad de vida de las personas y del ambiente (Arroyave, Builes \& Jaramillo, 2012).

De acuerdo con Perevochtchikova (2010), se concibe la cultura ambiental como el conjunto o sistema general de creencias, valores, actitudes y comportamientos de los integrantes de una sociedad que inciden en la transformación de la relación entre la sociedad y la naturaleza, asumiendo la responsabilidad social presente y futura. En esta perspectiva, se plantea una reconstrucción de la concepción del mundo, repensando, la significación y simbolismos asignados por la cultura, a la naturaleza (Leff, 2005).

El II Congreso Iberoamericano de Educación Ambiental, desarrollado en 1997, arrojo algunas conclusiones como que se reconoce la dificultad de aplicar enfoques interdisciplinarios en la práctica y la insuficiente correspondencia entre esta última y los postulados que se enuncian en la planificación de la comunicación y la educación ambiental. La comunicación en función del desarrollo local y la educación que genere una cultura ambiental en las comunidades, comienzan llamando la atención por su entorno natural, para modificar las actitudes que pueden dañar ese espacio común.

\section{Proyecto Ambiental Escolar - PRAE}

La responsabilidad de la comunidad educativa frente al diseño y desarrollo del PRAE quedo establecida en el decreto 1473 de 1994, reglamentario de la Ley General de Educación (Ministerio de Educación Nacional (MEN), 1994) el cual exige a todas las escuelas, tanto oficiales como privados, del país introducir en su currículo la dimensión ambiental y desarrollar un proyecto ambiental escolar de acuerdo con los principios rectores de interculturalidad, formación en valores, regionalización, de interdisciplinaridad y de participación compartida en el diseño y desarrollo del PRAE. Con alcance a todos los niveles de educación formal y establece los mecanismos de coordinación entre el MEN y el Ministerio de Ambiente y Desarrollo Sostenible.

Por lo tanto, es importante generar un sentido crítico y reflexivo de la comunidad donde se desarrolla permitiendo identificar la coherencia entre la educación ambiental impartida hoy y la visión o enfoque de desarrollo sostenible formulado en la agenda ambiental o planes de desarrollo municipal.

Según Leff (1998), no ha sido fácil transitar del pensamiento y los métodos de la complejidad, hacia el diseño y conducción de programas interdisciplinarios de formación que sea algo más que la simple conjunción de disciplinas para armar un programa multitemático.
Según Leff (1998), no ha sido fácil transitar del pensamiento y los métodos de la complejidad, hacia el diseño y conducción de programas interdisciplinarios de formación que sea algo más que la simple conjunción de disciplinas para armar un programa multitemático. 
La enseñanza interdisciplinaria en el campo ambiental implica la construcción de nuevos saberes, técnicas y conocimientos y su incorporación como contenidos integrados en el proceso de formación. Ello requiere un proceso de autoformación y la formación colectiva del equipo de enseñantes, de acotamiento de diversas temáticas ambientales, de elaboración de estrategias docentes y definición de nuevas estructuras curriculares

El concepto de eje transversal dentro del currículo se refiere a que la enseñanza debe estar presente como un conjunto amplio de componentes conceptuales, hábitos, destrezas y actitudes que atraviesan la estructura general de las distintas asignaturas, incorporando conexiones entre ellas y aportando el concepto de trabajo interdisciplinario (Illera, 1999).

Los PRAE, se pueden entender como proyectos transversales, que desde la institución escolar, se juntan a la exploración de alternativas de solución de una problemática $\mathrm{y} / \mathrm{o}$, al reconocimiento de potencialidades ambientales particulares locales, regionales y/o nacionales, lo cual les permite generar espacios comunes de reflexión, para el conocimiento significativo, para el desarrollo de criterios de solidaridad, tolerancia (respeto a la diferencia), búsqueda del consenso y autonomía; preparando para la gestión, desde una concepción de sostenibilidad ambiental (Torres, 1992).

De acuerdo con Torres (1992) el perfil de un PRAE debe estar inmerso en el PEI institucional, debe contener introducción, problema ambiental, actividades enfocadas al fortalecimiento de competencias de pensamiento científico y ciudadanas para comprender interacciones entre naturaleza sociedad y cultura. Igualmente es necesario tener una visión pedagógica para construir el conocimiento científico, espacios o mecanismos operativos para el dialogo de saberes, así mismo se debe observar el trabajo interdisciplinario de la comunidad educativa y actores de carácter local, regional, departamental y nacional.

Los procesos de investigación que se impulsan a través del PRAE, deben permitir identificar algunos elementos conceptuales, metodológicos y estratégicos de desarrollo de la educación ambiental y lograr incidir en la transformación de la institución educativa, desde el diálogo permanente con la sociedad, en la que se contempla el desafío de integrar las visiones sociales, culturales, políticas, económicas y ecológicas en los procesos de aprendizaje hacia una responsabilidad individual y colectiva (Orellana, 2005).

\section{Incorporación de la educación ambiental en la escuela}

Con respecto a la educación ambiental, un tema moderno que atañe a todos y debe llegar hasta el más pequeño de la población, por esto hay que estimular campañas de sensibilización y concienciación ambiental en los ciudadanos, para enriquecer esa dimensión ambiental en nuestra 
población y la mejor manera es desde las aulas de clase donde se están formando los adultos del mañana. La educación ambiental debe ir dirigida a la solución de la problemática ambiental teniendo en cuenta la relación que existe entre las personas y el entorno que no se limita únicamente a considerar el entorno como el espacio físico donde se da una conducta determinada (Rengifo, 2012).

Los educandos deben vivir experiencias significativas, mitigando en forma grupal o individual problemas ambientales de su escuela o comunidad, porque la educación ambiental (EA) requiere un cambio en las conductas y valores de la población con respecto del medio que le rodea.

Navarro \& Garrido (2006) mencionan que la escuela, en su labor pedagógica, debe poner en contacto al estudiante con el medio natural y los problemas ambientales de su escuela-comunidad, es decir, ofrecerles la experiencia a través de una serie de actividades encaminadas a la conservación del entorno.

Corral (1998) afirma que no basta con poseer conocimientos sobre estrategias de acción, es necesario adquirir habilidades instrumentales que permitan cuidar el entorno de manera sistemática y efectiva. Tilbury (1995, citado en García, 2003) menciona que una EA orientada hacia la acción debe involucrar a los estudiantes en el tratamiento de problemas reales y concretos, sin conformarse con la mera discusión de posibles soluciones. Se trata de prepararse para la acción ambiental, de adquirir capacidades generales, habilidades y rutinas concretas mediante faenas de mejora del paisaje. Los alumnos no sólo deben saber valorar, sino estar preparados y capacitados para la prevención y conservación del medio por otro lado la solución de problemas ambientales.

\section{Metodología}

El tipo de investigación fue corte cualitativo, con una orientación comprensiva, porque está encaminada a develar los sentidos y propone como alternativas para el análisis la información derivada de las técnicas e instrumentos de investigación frente a la comprensión y vivencia de lo ambiental y lo educativo ambiental.

El método de investigación utilizado fue la investigación acción, propuesta por Elliott (1993), que la define como un estudio de una situación social con el fin de mejorar la calidad de la acción dentro de la misma.

La población general para el estudio está conformada por una institución educativa integrada por 659 estudiantes, de los grados preescolar, básica primaria, y media, con un promedio de 28 estudiantes por grupo, la gran mayoría de ellos son de estrato uno y dos del SISBEN. Provenientes de diferentes sectores de la ciudad de Duitama (Boyacá), en especial de los alrededores de la institución. Específicamente se está trabajando con los estudiantes del grado quinto, algunos de ellos con necesidades educativas y con los estudiantes de grado sexto, con edades que oscilan entre los 10 y 15 años. De igual forma se 
aplicaran instrumentos de recolección de datos a 28 docentes, dos directivos docentes y 8 administrativos.

Dado el contexto social de esta investigación se considera la encuesta como un instrumento para la descripción y la predicción de un fenómeno (Bizquerra, 2004:233), este tipo de estudios permiten recoger información de individuos de diferentes formas y pretenden hacer estimaciones de las conclusiones a la población de referencia.

\section{Resultados}

\section{Observación directa e indagación}

Análisis del PEI - currículo En la institución las ciencias naturales son concebidas como una materia de amplio aprendizaje conceptual y que se puede integrar a otras áreas a través de proyectos pero falta el desarrollo de competencias científicas en los estudiantes. En el PEI está establecido el proceso de formación en valores basado en el respeto y cuidado del medio ambiente con todos sus elementos.

Análisis de las clases Aunque se pretende un aprendizaje significativo en las clases no se alcanza este objetivo pues son demasiados los contenidos y el tiempo no es suficiente. El docente explica y el estudiante escucha sin poder debatir sobre los temas; por lo tanto, los estudiantes son solo receptores de la información. Los estudiantes no hacen aportes y tampoco preguntan. La estrategia usada por los docentes es la exposición. Los estudiantes presentan desinterés y poca participación, además realizan actividades totalmente diferentes a las sugeridas por la docente y prefieren realizar dibujos libres, escribir cartas y jugar con los celulares.

Se conserva una pedagogía tradicional, no se fomenta la interacción de conocimientos ni retroalimentación, no hay flexibilidad en el aprendizaje ni trabajo en equipo, falta apropiación para fortalecer el proceso con actitudes intereses, no se fortalecen las competencias básicas como comprender, leer, escribir, escuchar, realizar operaciones básicas, plantear y solucionar problemas e inducir el pensamiento científico y crítico.

Análisis impacto ambiental en la institución. El asunto ambiental al que se le ha dado mayor prioridad ha sido el del manejo de residuos sólidos institucionales, este se hace de manera inadecuada ya que no se hace una disposición de acuerdo a la normatividad, las zonas de recolección de basura no están demarcadas y por lo tanto se observa basura en varios sitios lo que lleva a la propagación de roedores e insectos, además de malos olores. En las oficinas no se llevan a cabo prácticas de ahorro del papel. Existe un gasto innecesario de agua ya que se presentan fugas. En los salones se prenden los bombillos de manera innecesaria por lo tanto el gasto de energía es elevado. Existe un deterioro paisajístico que afecta las funciones de los estudiantes y la comunidad educativa. Los estudiantes no contribuyen a alzar la basura y depositarla en el lugar correspondiente. 
Se aplicó una encuesta a docentes de acuerdo a las categorías ambiente y PRAE en donde se pudo apreciar que no existe una apropiación correcta por parte de los docentes sobre el tema ambiental, por tanto el desarrollo de la educación ambiental es poco significativa ya que no se tiene claridad en el concepto por lo que no se ha interiorizado y no se pone en práctica.

En cuanto a la encuesta a estudiantes se estableció que requieren un acompañamiento más ambiental en su formación para contribuir a su desarrollo y de esta manera apropiarse de los espacios en los cuales se desenvuelven para que sean promotores y formadores ambientales.

Existe poca conciencia, responsabilidad y respeto por parte de los estudiantes de las causas que afectan el equilibrio natural por lo que se requiere comprender las relaciones de interdependencia con el entorno a partir de reflexiones y generar actitudes de valoración y respeto hacia el medio ambiente. La cultura del reciclaje en los hogares de los estudiantes y en el colegio, se ve afectada por los malos hábitos, donde no se recolectan las basuras y residuos en los recipientes dispuestos para ello, afectando el ambiente y el mismo paisaje de forma desagradable; se encuentran papeles, plásticos, vidrios $\mathrm{y}$ otros residuos en sitios inadecuados, como prados, vías peatonales, aulas de clase y zonas deportivas.

Se puede apreciar la ausencia frente al concepto de ambiente ya que se enfoca más hacia las ciencias naturales pero no se manifiesta la idea amplia del concepto, se ve reducida y enfocada a los componentes de la naturaleza y los problemas de contaminación relacionados con la disposición final de los residuos sólidos y el ahorro de la energía y el agua. La idea o creencia sobre el ambiente está ligada a los eventos de la naturaleza. No se reconocen los proyectos ambientales que maneja el colegio como el propósito y la manera en que funciona $y$ no se demuestra interés por conocerlo a fondo.

No existe una apropiación correcta por parte de los docentes sobre el tema del ambiente no se puede lograr una educación ambiental significativa ya que no se tiene claridad en el concepto por lo que no se ha interiorizado y no se practica.

\section{Triangulación de la información}

Para los estudiantes educación ambiental es cuidar el medio ambiente y educar; mientras que para los docentes es formación y concientización. Es de anotar que la educación ambiental debe girar en torno a un proceso de conciencia para fortalecer actitudes y valores y desarrollar habilidades para la protección ambiental. Es necesario que tanto docentes como estudiantes enfoquen la enseñanza y aprendizaje hacia la formación de consciencia.

Para los estudiantes los temas que se pueden abordar desde la educación ambiental son arborizar cuidar el medio ambiente; mientras que para los docentes las temáticas a tener en cuenta son la preservación, concientización
Para los estudiantes educación ambiental es cuidar el medio ambiente y educar; mientras que para los docentes es formación y concientización. 
y reciclaje. Es de anotar que en la enseñanza - aprendizaje de educación ambiental se deben abarcar múltiples dimensiones asumiéndolo desde lo conceptual pero también desde la práctica e integrando dimensiones como la motivación hacia la acción.

Los estudiantes frente a las problemáticas ambientales creen que su papel es recoger basura, no contaminar, decirle a la gente que no contamine, cuidar el ambiente, mientras que para los docentes sus responsabilidades se enfocan hacia concientizar, enseñar y realizar campañas. Desde la escuela se deben fortalecer las orientaciones teóricas y metodológicas pasando a la práctica vinculando a docentes y estudiantes frente a su realidad geográfica y ambiental, convirtiendo la actividad pedagógica en un aliado para la participación activa y reflexiva de los estudiantes frente a temas ambientales. Desde su práctica, el docente enriquece los valores, posibilita el conocimiento, estimula las habilidades y contribuye al desarrollo de las capacidades del individuo.

Entre las asignaturas que los estudiantes abordarían para los problemas ambientales están naturales y talleres, mientras que los docentes dicen que buscarían origen, causas y consecuencias. Los docentes tendrían en cuenta la formación, reciclaje y apoyo. Es de aclarar que tanto para abordar aspectos que aborden soluciones y las asignaturas para solucionar problemas lo más importante es tener en cuenta la comunicación en función del desarrollo local y la educación que genere una cultura ambiental en las comunidades, comienzan llamando la atención por su entorno natural, para modificar las actitudes que pueden dañar ese espacio común.

Los estudiantes frente al apoyo del PRAE dicen que lo deben dar los profesores, seguido de todos; para los docentes promover la educación ambiental en la comunidad educativa depende de todos los actores de la escuela y con el mismo nivel de participación los docentes, seguido del rector, estudiantes y los docentes de todas las áreas y niveles. En ambas circunstancias se nota como los docentes son quienes están llamados a promover la educación ambiental y en este caso apoyar el PRAE en la institución educativa, pero promover la educación ambiental debe ser responsabilidad de toda la comunidad educativa.

Los estudiantes creen que la educación ambiental es una alternativa para mejorar las problemáticas ambientales, dando razones como el cuidado del medio ambiente; para los docentes los aportes que se han generado han sido desde el reciclaje y la concientización, pero no hablan de las actividades o participación frente al PRAE desde una perspectiva socio-cultural y abordaje amplio desde la visión del ambiente como sistema, que implica la comprensión no solo de los sistemas naturales, sino también de los sistemas sociales, culturales, ecológicos, políticos y tecnológicos, entre otros.

Los estudiantes relacionan como acciones del PRAE algunas situaciones en las que han participado en la siembra 
de árboles y otros sencillamente lo acogen desde emprender algunas actividades para cuidar el medio ambiente, situación que denota la ausencia de procesos constantes y permanentes de educación ambiental $y$ en la que el estudiante se visualice como actor clave que participa y propone y no solo quien es espectador o actor pasivo. En esta situación los docentes no identifican el PRAE como estrategia para dinamizar la Educación Ambiental por lo que se desarrollan actividades desarticuladas y aisladas a los propósitos de este tipo de proyectos.

En este sentido se espera que la institución educativa responda a las necesidades que plantean los contextos locales desde su PRAE, pero también a los retos y problemáticas generadas por un mundo globalizado que ha dado lugar a una sociedad de consumo donde priman los intereses económicos, lo cual ha consolidado prácticas que ponen en riesgo el bienestar e incluso la existencia de la vida humana.

De acuerdo con los antecedentes revisados, en la actualidad existe falta de conciencia, responsabilidad y respeto por el ambiente, hecho que afecta el equilibrio socio-natural, de esta manera es fundamental ser parte de la solución y reflexionar sobre la importancia de la educación ambiental, donde se promueva la comprensión de las interrelaciones del hombre con su entorno y con ello se motive al conocimiento reflexivo y crítico de la realidad.

Desde los alcances planteados de la Educación Ambiental en el Instituto Técnico La Nueva Familia se presenta toda una ruta para explorar y desarrollar iniciativas que permitan fomentar capacidades y sensibilidades en torno a la dimensión ambiental y con ello que la comunidad educativa se comprometa con su PRAE como estrategia que une e integra saberes, prácticas y experiencias de todos los actores locales en pro de reconocer su entorno y sobre él comprender su realidad para formar individuos con toda la disposición para generar transformaciones desde el ser y el hacer, situación que permitirá visibilizar una verdadera cultura ambiental.

\section{Referencias}

Ángel A. (1992). Perspectivas pedagógicas en la educación ambiental. Una visión interdisciplinaria. Colombia.

Arango, P. (2015). Sentidos de la Educación Ambiental para las Formadoras de la Primera Infancia en el Núcleo Educativo 915 de Medellín Universidad de Manizales Facultad de ciencias contables, económicas y administrativas Maestría en Desarrollo Sostenible y Medio Ambiente. Cohorte vii Manizales, Colombia. 
Arroyave, J., Builes, L., \& Rodríguez, E. (2012). La Gestión Socio-Ambiental y el Recurso Hídrico. Journal of Engineering and technology. 1(1), 62-70.. Recuperado de: http://repository.lasallista.edu.co,

Barrera, C. (2014). Determinación del nivel de conocimiento ambiental de los estudiantes de básica secundaria y media en las instituciones educativas públicas del municipio de Manizales. Universidad de Manizales facultad de ciencia contables, económicas y administrativas, centro de investigaciones en medio ambiente y desarrollo, maestría en desarrollo sostenible y medio ambiente Manizales.

Bizquerra, R. (2003). Metodología de la investigación educativa. La Muralla.

Calvo, S. (1997) Redefinir la Educación Ambiental de las Recomendaciones de Tibilisi a la Agenda XXI. Ministerio del Medio Ambiente. Madrid.

Caride \& Meira: (2001). Educación ambiental y desarrollo bumano. Ariel Educación. Barcelona.

Corral, V. (1998) Aportes de la Psicología ambiental en pro de una conducta ecológica responsable, en: Guevara, Javier et. al. (Coord.): Estudios de Psicología Ambiental en América Latina, México: UNAM, CONACYT, UAP, pp. 71-95

Da Silva, T. (2001) Espacios de identidad. Nuevas visiones sobre el currículum.

Duque, (2015) Educación ambiental de estudiantes, docentes y directivas de educación básica secundaria. Maestría en desarrollo sostenible y medio ambiente cimad centro de investigaciones en medio ambiente y desarrollo Pereira.

Elliott, J. (1993). El cambio educativo desde la investigación-acción, Madrid: Morata.

García, M. (1993). La encuesta. En: García M, Ibáñez J, Alvira F. El análisis de la realidad social. Métodos y técnicas de Investigación. Madrid: Alianza Universidad Textos; p. 141-70.

Illera T. (1999). Cia Ltda Manual Práctico de PRAES (1999)

Leff, E. (1989). Saber ambiental, sustentabilidad, racionalidad, complejidad, poder. México.

Navarro, S., \& Garrido, J. (2006) significado del cuidado ambiental: un estudio de caso en educación secundaria REICE. Revista Iberoamericana sobre Calidad, Eficacia y Cambio en Educación, 4, (1), pp. 52-70.

Orellana, I. (2005). La estrategia pedagógica de comunidad de aprendizaje, definiendo sus fundamentos, sus prácticas y su pertinencia en la educación ambiental, 2005. 
Perevochtchikova, M. (2010). Nueva cultura del agua en México: avances, limitaciones y retos. Revista Latinoamericana de recursos naturales, Recuperado de: http://www.itson.mx/publicaciones/ rlrn/Documents/v6-n2-2-nuevacultura-del-aguaen-mexico.pdf.

Pérez, P. (2013). Comportamiento ambiental en estudiantes de secundaria. Recuperado de Revista Vinculando: http://vinculando.org/educacion/comportamientoambiental-en-estudiantes-de-secundaria.html

Proyecto Educativo Institucional, Institución Educativa Colegio la Nueva Familia, Duitama 2016.

Reguillo, R. (2012). Pensar la cultura con y después de Bourdieu. Revista Universidad de Guadalajara. México. 2001. Recuperado de: http://www.cge. udg.mx/ revistaudg/rug24/bourdieu4.html

Rengifo, B., Quitiaquez, L. \& Mora, F. (2012). La educación ambiental una estrategia pedagógica que contribuye a la solución de la problemática ambiental en Colombia. XII Coloquio internacional de Geocrítica. Colombia, 2012.

Rondón, I. (2016) Percepciones socio ambientales en las comunidades estudiantiles de dos instituciones educativas dentro del contexto rural y urbano, hacia el PNN ranchería en Paipa Boyacá, a través de educación ambiental.

Torres M, (1992). Dimensión ambiental. Un reto para la educación ambiental. Colombia, 1992.

Torres, I. (2011), Medio ambiente y Proyecto Ambiental Escolar (PRAE) en el Colegio Nicolás Esguerra. Universidad Nacional de Colombia Facultad de Ciencias Bogotá, Colombia 2011 